\title{
Existence theory for fractional differential equations with non-separated type nonlocal multi-point and multi-strip boundary conditions
}

\author{
Bashir Ahmad $^{1 *}$ (D, Sotiris K. Ntouyas ${ }^{1,2}$, Ahmed Alsaedi ${ }^{1}$, Wafa Shammakh¹ and Ravi P. Agarwal ${ }^{3}$
}

"Correspondence:

bashirahmad_qau@yahoo.com

'NAAM-Research Group,

Department of Mathematics,

Faculty of Science, King Abdulaziz

University, Jeddah, Saudi Arabia

Full list of author information is

available at the end of the article

\begin{abstract}
We introduce a more general class of fractional-order boundary value problems involving non-separated type multi-point and multi-strip boundary conditions. Several existence and uniqueness results for the given problem are established by applying the tools of fixed-point theory. Some illustrative examples are also included. The boundary conditions introduced in this work are of quite general nature and reduce to many special cases by fixing the parameters involved in the conditions.
\end{abstract}

MSC: 26A33; 34A08; 34B15

Keywords: Caputo fractional derivative; Nonlocal; Multi-point; Multi-strip; Boundary conditions

\section{Introduction}

In the last few decades, fractional-order differential equations equipped with a variety of boundary conditions have been studied. The literature on the topic includes the existence and uniqueness results related to classical, periodic/anti-periodic, nonlocal, multi-point, and integral boundary conditions, for instance, see [1-18].

In this paper, motivated by the aforementioned works, we consider a more general class of boundary value problems of Caputo type fractional differential equations and nonseparated type multi-point and multi-strip boundary conditions. Precisely, we investigate the existence and uniqueness of solutions for the following fractional differential equation:

$$
{ }^{c} D^{q} x(t)=f\left(t, x(t),{ }^{c} D^{\beta} x(t)\right), \quad 0<\beta<1,1<q \leq 2, t \in[0,1],
$$

supplemented with the boundary conditions of the form:

$$
\left\{\begin{array}{l}
a x(0)+b x(1)=\sum_{i=1}^{m-2} \alpha_{i} x\left(\sigma_{i}\right)+\sum_{j=1}^{p-2} r_{j} \int_{\xi_{j}}^{\eta_{i}} x(s) d s, \\
c x^{\prime}(0)+d x^{\prime}(1)=\sum_{i=1}^{m-2} \delta_{i} x^{\prime}\left(\sigma_{i}\right)+\sum_{j=1}^{p-2} \gamma_{j} \int_{\xi_{j}}^{\eta_{j}} x^{\prime}(s) d s, \\
0<\sigma_{1}<\sigma_{2}<\cdots<\sigma_{m-2}<\cdots<\xi_{1}<\eta_{1}<\xi_{2}<\eta_{2}<\cdots<\xi_{p-2}<\eta_{p-2}<1,
\end{array}\right.
$$

where ${ }^{c} D^{q},{ }^{c} D^{\beta}$ denote the Caputo fractional derivatives of order $q$ and $\beta$, respectively,

(c) The Author(s) 2018. This article is distributed under the terms of the Creative Commons Attribution 4.0 International License (http://creativecommons.org/licenses/by/4.0/), which permits unrestricted use, distribution, and reproduction in any medium, provided you give appropriate credit to the original author(s) and the source, provide a link to the Creative Commons license, and indicate if changes were made. 
$f$ is a given continuous function, $a, b, c, d$ are real constants, and $\alpha_{i}, \delta_{i}(i=1,2, \ldots, m-$ 2), $r_{j}, \gamma_{j}(j=1,2, \ldots, p-2)$ are positive real constants.

Here we remark that the boundary conditions (1.2) can be interpreted in the sense that the linear combinations of values of the unknown function and its derivative at the end points of the interval under consideration are proportional to the sum of their respective multi-point and multi-strip values. It is imperative to mention that nonlocal boundary conditions play a key role in formulating physical, chemical, or other processes involving some peculiarities occurring inside the domain, for instance, see [19]; while the integral boundary conditions find useful applications in the computational fluid dynamics (CFD) studies of blood flow [20] and regularization of ill-posed parabolic backward problems in time partial differential equations (e.g., mathematical models for bacterial self-regularization [21]).

The rest of the paper is organized as follows. We recall some basic concepts of fractional calculus and introduce the integral operator associated with the given problem in Sect. 2. Existence results, which rely on Schauder's fixed point theorem, nonlinear alternative for single valued maps, and Krasnoselskii's fixed point theorem, are presented in Sect. 3. In Sect. 4, we obtain the uniqueness results by means of Boyd and Wong's fixed point theorem and Banach's fixed point theorem together with Hölder's inequality. Examples illustrating the obtained results are presented in Sect. 5 , and the paper concludes with some interesting observations in Sect. 6.

\section{Preliminaries}

First of all, we recall some basic definitions [22]. For $\alpha>0,[\alpha]$ denotes the integer part of the real number $\alpha$.

Definition 2.1 The Riemann-Liouville fractional integral of order $\alpha>0$ for a locally integrable real-valued function $f$ on $0 \leq t<b<\infty$ is defined as

$$
I^{\alpha} y(t)=\frac{1}{\Gamma(\alpha)} \int_{0}^{t}(t-s)^{\alpha-1} y(s) d s
$$

Definition 2.2 Let $f \in L^{1}[a, b],-\infty \leq a<t<b \leq+\infty$ and $f * K_{n-\alpha} \in W^{n, 1}[a, b], n=[\alpha]+1$, $\alpha>0$, where $W^{n, 1}[a, b]$ is the Sobolev space defined as

$$
W^{n, 1}[a, b]=\left\{f \in L^{1}[a, b]: \frac{d^{n}}{d t^{n}} f \in L^{1}[a, b]\right\} .
$$

The Riemann-Liouville fractional derivative $D_{a}^{\alpha}$ of order $\alpha>0(n-1<\alpha<n, n \in \mathbb{N})$ is defined as

$$
D_{a}^{\alpha} f(t)=\frac{d^{n}}{d t^{n}} I_{a}^{1-\alpha} f(t)=\frac{1}{\Gamma(n-\alpha)} \frac{d^{n}}{d t^{n}} \int_{a}^{t}(t-s)^{n-1-\alpha} f(s) d s .
$$

Definition 2.3 If $f \in C^{n}[a, b]$, then the Caputo fractional derivative ${ }^{c} D_{a}^{\alpha}$ of order $\alpha \in \mathbb{R}$ $(n-1<\alpha<n, n \in \mathbb{N})$ is defined as

$$
{ }^{c} D_{a}^{\alpha} f(t)=I_{a}^{1-\alpha} f^{(n)}(t)=\frac{1}{\Gamma(n-\alpha)} \int_{a}^{t}(t-s)^{n-1-\alpha} f^{(n)}(s) d s .
$$

In passing we remark that the Caputo fractional derivative ${ }^{c} D_{a}^{\alpha}$ with $a=0$ is denoted by ${ }^{c} D^{\alpha}$. 
Remark 2.4 (see [22, 23]) (i) If $\alpha>0, \beta>0, f \in L(0,1)$, then

$$
I^{\alpha} I^{\beta} f(t)=I^{\alpha+\beta} f(t), \quad D^{\alpha} I^{\alpha} f(t)=f(t), \quad D^{\alpha} I^{\beta} f(t)=I^{\beta-\alpha} f(t) .
$$

(ii) $I^{\alpha} D^{\alpha} f(t)=f(t), 0<\alpha<1, f \in C([0,1])$, and $D^{\alpha} f \in C(0,1) \cap L(0,1)$.

Next, we present an auxiliary lemma which plays a key role in the sequel.

Lemma 2.5 For $g \in C[0,1]$, the solution of the linear fractional differential equation

$$
{ }^{c} D^{q} x(t)=g(t), \quad 1<q \leq 2, t \in[0,1],
$$

subject to the boundary conditions (1.2) is equivalent to the fractional integral equation

$$
\begin{aligned}
x(t)= & \int_{0}^{t} \frac{(t-s)^{q-1}}{\Gamma(q)} g(s) d s+\frac{\left(\Delta_{2} t-\Delta_{3}\right)}{\Delta_{1} \Delta_{2}}\left[\sum_{i=1}^{m-2} \delta_{i} \int_{0}^{\sigma_{i}} \frac{\left(\sigma_{i}-s\right)^{q-2}}{\Gamma(q-1)} g(s) d s\right. \\
& \left.+\sum_{j=1}^{p-2} \gamma_{j} \int_{\xi_{j}}^{\eta_{j}}\left(\int_{0}^{s} \frac{(s-u)^{q-2}}{\Gamma(q-1)} g(u) d u\right) d s-d \int_{0}^{1} \frac{(1-s)^{q-2}}{\Gamma(q-1)} g(s) d s\right] \\
& +\frac{1}{\Delta_{2}}\left[\sum_{i=1}^{m-2} \alpha_{i} \int_{0}^{\sigma_{i}} \frac{\left(\sigma_{i}-s\right)^{q-1}}{\Gamma(q)} g(s) d s\right. \\
& \left.+\sum_{j=1}^{p-2} r_{j} \int_{\xi_{j}}^{\eta_{j}}\left(\int_{0}^{s} \frac{(s-u)^{q-1}}{\Gamma(q)} g(u) d u\right) d s-b \int_{0}^{1} \frac{(1-s)^{q-1}}{\Gamma(q)} g(s) d s\right],
\end{aligned}
$$

where

$$
\begin{aligned}
& \Delta_{1}=c+d-\mu_{1} \neq 0, \quad \Delta_{2}=a+b-\mu_{2} \neq 0, \quad \Delta_{3}=b-\mu_{3}, \\
& \mu_{1}=\sum_{i=1}^{m-2} \delta_{i}-\sum_{j=1}^{p-2} \gamma_{j}\left(\eta_{j}-\xi_{j}\right), \quad \mu_{2}=\sum_{i=1}^{m-2} \alpha_{i}-\sum_{j=1}^{p-2} r_{j}\left(\eta_{j}-\xi_{j}\right), \\
& \mu_{3}=\sum_{i=1}^{m-2} \alpha_{i} \sigma_{i}-\sum_{j=1}^{p-2} r_{j} \frac{\left(\eta_{j}^{2}-\xi_{j}^{2}\right)}{2} .
\end{aligned}
$$

Proof It is well known [22] that the solution of fractional differential equation (2.1) can be written as

$$
x(t)=\int_{0}^{t} \frac{(t-s)^{q-1}}{\Gamma(q)} g(s) d s+b_{0}+b_{1} t,
$$

where $b_{0}, b_{1} \in \mathbb{R}$ are arbitrary constants. Using the boundary conditions (1.2) together with (2.6), we find that

$$
\begin{aligned}
b_{1}= & \frac{1}{\Delta_{1}}\left[\sum_{i=1}^{m-2} \delta_{i} \int_{0}^{\sigma_{i}} \frac{\left(\sigma_{i}-s\right)^{q-2}}{\Gamma(q-1)} g(s) d s\right. \\
& \left.+\sum_{j=1}^{p-2} \gamma_{j} \int_{\xi_{j}}^{\eta_{j}}\left(\int_{0}^{s} \frac{(s-u)^{q-2}}{\Gamma(q-1)} g(u) d u\right) d s-d \int_{0}^{1} \frac{(1-s)^{q-2}}{\Gamma(q-1)} g(s) d s\right],
\end{aligned}
$$




$$
\begin{aligned}
b_{0}= & -\frac{\Delta_{3}}{\Delta_{2}} b_{1}+\frac{1}{\Delta_{2}}\left[\sum_{i=1}^{m-2} \alpha_{i} \int_{0}^{\sigma_{i}} \frac{\left(\sigma_{i}-s\right)^{q-1}}{\Gamma(q)} g(s) d s\right. \\
& \left.+\sum_{j=1}^{p-2} r_{j} \int_{\xi_{j}}^{\eta_{j}}\left(\int_{0}^{s} \frac{(s-u)^{q-1}}{\Gamma(q)} g(u) d u\right) d s-b \int_{0}^{1} \frac{(1-s)^{q-1}}{\Gamma(q)} g(s) d s\right],
\end{aligned}
$$

where $\Delta_{k}(k=1,2,3)$ are given by (2.3). Substituting the values given by (2.7) and (2.8) in (2.6), we obtain (2.2). The converse follows by direct computation. This completes the proof.

In the forthcoming analysis, we define the space

$$
X=\left\{x \mid x \in C([0,1], \mathbb{R}) \text { and }{ }^{c} D^{\beta} x \in C([0,1], \mathbb{R})\right\},
$$

equipped with the norm

$$
\|x\|_{X}=\sup _{t \in[0,1]}|x(t)|+\sup _{t \in[0,1]}\left|{ }^{c} D^{\beta} x(t)\right|
$$

where ${ }^{c} D^{\beta}$ denotes the standard Caputo fractional derivative of order $0<\beta \leq 1$. As shown in [24], $\left(X,\|\cdot\|_{X}\right)$ is a Banach space.

Introduce an operator $F: X \rightarrow X$ as follows:

$$
\begin{aligned}
F(x)(t)= & \int_{0}^{t} \frac{(t-s)^{q-1}}{\Gamma(q)} f\left(s, x(s),{ }^{c} D^{\beta} x(s)\right) d s \\
& +\frac{\left(\Delta_{2} t-\Delta_{3}\right)}{\Delta_{1} \Delta_{2}}\left[\sum_{i=1}^{m-2} \delta_{i} \int_{0}^{\sigma_{i}} \frac{\left(\sigma_{i}-s\right)^{q-2}}{\Gamma(q-1)} f\left(s, x(s),{ }^{c} D^{\beta} x(s)\right) d s\right. \\
& +\sum_{j=1}^{p-2} \gamma_{j} \int_{\xi_{j}}^{\eta_{j}}\left(\int_{0}^{s} \frac{(s-u)^{q-2}}{\Gamma(q-1)} f\left(u, x(u),{ }^{c} D^{\beta} x(u)\right) d u\right) d s \\
& \left.-d \int_{0}^{1} \frac{(1-s)^{q-2}}{\Gamma(q-1)} f\left(s, x(s),{ }^{c} D^{\beta} x(s)\right) d s\right] \\
& +\frac{1}{\Delta_{2}}\left[\sum_{i=1}^{m-2} \alpha_{i} \int_{0}^{\sigma_{i}} \frac{\left(\sigma_{i}-s\right)^{q-1}}{\Gamma(q)} f\left(s, x(s),{ }^{c} D^{\beta} x(s)\right) d s\right. \\
& +\sum_{j=1}^{p-2} r_{j} \int_{\xi_{j}}^{\eta_{j}}\left(\int_{0}^{s} \frac{(s-u)^{q-1}}{\Gamma(q)} f\left(u, x(u),{ }^{c} D^{\beta} x(u)\right) d u\right) d s \\
& \left.-b \int_{0}^{1} \frac{(1-s)^{q-1}}{\Gamma(q)} f\left(s, x(s),{ }^{c} D^{\beta} x(s)\right) d s\right],
\end{aligned}
$$

and observe that problem (1.1)-(1.2) has solutions if and only if operator (2.9) has fixed points. Furthermore, using the value of $F^{\prime}(x)$ given by

$$
F^{\prime}(x)(t)=\int_{0}^{t} \frac{(t-s)^{q-2}}{\Gamma(q-1)} f\left(s, x(s),{ }^{c} D^{\beta} x(s)\right) d s
$$




$$
\begin{aligned}
& +\frac{1}{\Delta_{1}}\left[\sum_{i=1}^{m-2} \delta_{i} \int_{0}^{\sigma_{i}} \frac{\left(\sigma_{i}-s\right)^{q-2}}{\Gamma(q-1)} f\left(s, x(s),{ }^{c} D^{\beta} x(s)\right) d s\right. \\
& +\sum_{j=1}^{p-2} \gamma_{j} \int_{\xi_{j}}^{\eta_{j}}\left(\int_{0}^{s} \frac{(s-u)^{q-2}}{\Gamma(q-1)} f\left(u, x(u),{ }^{c} D^{\beta} x(u)\right) d u\right) d s \\
& \left.-d \int_{0}^{1} \frac{(1-s)^{q-2}}{\Gamma(q-1)} f\left(s, x(s),{ }^{c} D^{\beta} x(s)\right) d s\right]
\end{aligned}
$$

in Definition (2.3), we get

$$
\begin{aligned}
{ }^{c} D^{\beta}(F x)(t)= & \int_{0}^{t} \frac{(t-s)^{-\beta}}{\Gamma(1-\beta)} F^{\prime}(x)(s) d s \\
= & \int_{0}^{t} \frac{(t-s)^{q-\beta-1}}{\Gamma(q-\beta)} f\left(s, x(s),{ }^{c} D^{\beta} x(s)\right) d s \\
& +\frac{1}{\Delta_{1}} \frac{t^{1-\beta}}{\Gamma(2-\beta)}\left[\sum_{i=1}^{m-2} \delta_{i} \int_{0}^{\sigma_{i}} \frac{\left(\sigma_{i}-s\right)^{q-2}}{\Gamma(q-1)} f\left(s, x(s),{ }^{c} D^{\beta} x(s)\right) d s\right. \\
& +\sum_{j=1}^{p-2} \gamma_{j} \int_{\xi_{j}}^{\eta_{j}}\left(\int_{0}^{s} \frac{(s-u)^{q-2}}{\Gamma(q-1)} f\left(u, x(u),{ }^{c} D^{\beta} x(u)\right) d u\right) d s \\
& \left.-d \int_{0}^{1} \frac{(1-s)^{q-2}}{\Gamma(q-1)} f\left(s, x(s),{ }^{c} D^{\beta} x(s)\right) d s\right] .
\end{aligned}
$$

For convenience, we set the notations:

$$
\begin{aligned}
\Omega= & \frac{1}{\Gamma(q+1)}+\max _{t \in[0,1]} \frac{\left|\Delta_{2} t-\Delta_{3}\right|}{\left|\Delta_{1} \Delta_{2}\right|}\left[\sum_{i=1}^{m-2}\left|\delta_{i}\right| \frac{\sigma_{i}^{q-1}}{\Gamma(q)}+\sum_{j=1}^{p-2} \frac{\left|\gamma_{j}\right|}{\Gamma(q+1)}\left|\eta_{j}^{q}-\xi_{j}^{q}\right|+\frac{|d|}{\Gamma(q)}\right] \\
& +\frac{1}{\left|\Delta_{2}\right|}\left[\sum_{i=1}^{m-2}\left|\alpha_{i}\right| \frac{\sigma_{i}^{q}}{\Gamma(q+1)}+\sum_{j=1}^{p-2} \frac{\left|r_{j}\right|}{\Gamma(q+2)}\left|\eta_{j}^{q+1}-\xi_{j}^{q+1}\right|+\frac{|b|}{\Gamma(q+1)}\right], \\
\Omega_{1}= & \frac{1}{\Gamma(q-\beta+1)} \\
& +\frac{1}{\left|\Delta_{1}\right|} \frac{1}{\Gamma(2-\beta)}\left[\sum_{i=1}^{m-2}\left|\delta_{i}\right| \frac{\sigma_{i}^{q-1}}{\Gamma(q)}+\sum_{j=1}^{p-2} \frac{\left|\gamma_{j}\right|}{\Gamma(q+1)}\left|\eta_{j}^{q}-\xi_{j}^{q}\right|+\frac{|d|}{\Gamma(q)}\right], \\
Z_{1}= & \frac{1}{\Gamma(q)}\left(\frac{1-\gamma}{q-\gamma}\right)^{1-\gamma}+\max _{t \in[0,1]} \frac{\left|\Delta_{2} t-\Delta_{3}\right|}{\left|\Delta_{1} \Delta_{2}\right|} \frac{1}{\Gamma(q-1)}\left(\frac{1-\gamma}{q-\gamma-1}\right)^{1-\gamma}\left[\sum_{i=1}^{m-2}\left|\delta_{i}\right|\right. \\
& \left.+\sum_{j=1}^{p-2}\left|\gamma_{j}\right| \frac{\eta^{q-\gamma}-\xi^{q-\gamma}}{q-\gamma}+|d|\right]+\frac{1}{\left|\Delta_{2}\right|} \frac{1}{\Gamma(q)}\left(\frac{1-\gamma}{q-\gamma}\right)^{1-\gamma}\left[\sum_{i=1}^{m-2}\left|\alpha_{i}\right|\right. \\
& \left.\left.+\sum_{j=1}^{p-2}\left|r_{j}\right| \frac{\eta^{q-\gamma+1}-\xi^{q-\gamma+1}}{q-\gamma+1}+|b|\right], \frac{\gamma \in(0, q-1)}{\gamma}\right] \\
& =1
\end{aligned}
$$


and

$$
\begin{aligned}
Z_{2}= & \frac{1}{\Gamma(q-\beta)}\left(\frac{1-\gamma}{q-\beta-\gamma}\right)^{1-\gamma}+\frac{1}{\left|\Delta_{1}\right|} \frac{1}{\Gamma(2-\beta)}\left(\frac{1-\gamma}{q-\gamma-1}\right)^{1-\gamma}\left[\sum_{i=1}^{m-2}\left|\delta_{i}\right|\right. \\
& \left.+\sum_{j=1}^{p-2}\left|\gamma_{j}\right| \frac{\eta^{q-\gamma}-\xi^{q-\gamma}}{q-\gamma}+|d|\right] .
\end{aligned}
$$

\section{Existence results}

This section is devoted to the existence results for problem (1.1)-(1.2). Our first existence result is based on Schauder's fixed point theorem.

Lemma 3.1 (Schauder's fixed point theorem [25]) Let $U$ be a closed, convex, and nonempty subset of a Banach space $X$. Let $P: U \rightarrow U$ be a continuous mapping such that $P(U)$ is a relatively compact subset of $X$. Then $P$ has at least one fixed point in $U$.

Theorem 3.2 Assume that $f:[0,1] \times \mathbb{R} \times \mathbb{R} \rightarrow \mathbb{R}$ is a continuous function satisfying the assumption:

$\left(A_{1}\right)|f(t, x, y)| \leq m(t)+d_{1}|x|^{\rho_{1}}+d_{2}|y|^{\rho_{2}}, \forall(t, x, y) \in[0,1] \times \mathbb{R} \times \mathbb{R}$, and $m \in L^{\frac{1}{\gamma}}\left([0,1], \mathbb{R}^{+}\right)$, $\gamma \in(0, q-1), d_{i} \geq 0,0 \leq \rho_{i}<1, i=1,2$.

Then the boundary value problem (1.1)-(1.2) has at least one solution on [0,1].

Proof Denote $\|m\|=\left(\int_{0}^{1}|m(s)|^{\frac{1}{\gamma}} d s\right)^{\gamma}$. Let $B_{r}=\left\{x \in X:\|x\|_{X} \leq r\right\}$ with $r>0$ to be specified later. It is clear that $B_{r}$ is a closed, bounded, and convex subset of the Banach space $X$.

We will show that there exists $r>0$ such that the operator $F$ maps $B_{r}$ into $B_{r}$. For $x \in B_{r}$, we have

$$
\begin{aligned}
|(F x)(t)| & \leq \int_{0}^{t} \frac{(t-s)^{q-1}}{\Gamma(q)}\left[m(s)+d_{1}|x(s)|^{\rho_{1}}+\left.\left.d_{2}\right|^{c} D^{\beta} x(s)\right|^{\rho_{2}}\right] d s \\
& +\max _{t \in[0,1]} \frac{\left|\Delta_{2} t-\Delta_{3}\right|}{\left|\Delta_{1} \Delta_{2}\right|}\left[\sum_{i=1}^{m-2}\left|\delta_{i}\right| \int_{0}^{\sigma_{i}} \frac{\left(\sigma_{i}-s\right)^{q-2}}{\Gamma(q-1)}\left[m(s)+d_{1}|x(s)|^{\rho_{1}}+\left.\left.d_{2}\right|^{c} D^{\beta} x(s)\right|^{\rho_{2}}\right] d s\right. \\
& +\sum_{j=1}^{p-2}\left|\gamma_{j}\right| \int_{\xi_{j}}^{\eta_{j}}\left(\int_{0}^{s} \frac{(s-u)^{q-2}}{\Gamma(q-1)}\left[m(u)+d_{1}|x(u)|^{\rho_{1}}+\left.\left.d_{2}\right|^{c} D^{\beta} x(u)\right|^{\rho_{2}}\right] d u\right) d s \\
& \left.+|d| \int_{0}^{1} \frac{(1-s)^{q-2}}{\Gamma(q-1)}\left[m(s)+d_{1}|x(s)|^{\rho_{1}}+\left.\left.d_{2}\right|^{c} D^{\beta} x(s)\right|^{\rho_{2}}\right] d s\right] \\
& +\frac{1}{\left|\Delta_{2}\right|}\left[\sum_{i=1}^{m-2}\left|\alpha_{i}\right| \int_{0}^{\sigma_{i}} \frac{\left(\sigma_{i}-s\right)^{q-1}}{\Gamma(q)}\left[m(s)+d_{1}|x(s)|^{\rho_{1}}+\left.\left.d_{2}\right|^{c} D^{\beta} x(s)\right|^{\rho_{2}}\right] d s\right. \\
& +\sum_{j=1}^{p-2} r_{j} \int_{\xi_{j}}^{\eta_{j}}\left(\int_{0}^{s} \frac{(s-u)^{q-1}}{\Gamma(q)}\left[m(u)+d_{1}|x(u)|^{\rho_{1}}+\left.\left.d_{2}\right|^{c} D^{\beta} x(u)\right|^{\rho_{2}}\right]\right) d s \\
& \left.+|b| \int_{0}^{1} \frac{(1-s)^{q-1}}{\Gamma(q)}\left[m(s)+d_{1}|x(s)|^{\rho_{1}}+d_{2}\left|{ }^{c} D^{\beta} x(s)\right|^{\rho_{2}}\right] d s\right]
\end{aligned}
$$


Ahmad et al. Advances in Difference Equations ( 2018) 2018:89

Page 7 of 20

$$
\begin{aligned}
\leq & \frac{\|m\|}{\Gamma(q)}\left(\frac{1-\gamma}{q-\gamma}\right)^{1-\gamma}+\frac{d_{1} r^{\rho_{1}}+d_{2} r^{\rho_{2}}}{\Gamma(q+1)} \\
& +\max _{t \in[0,1]} \frac{\left|\Delta_{2} t-\Delta_{3}\right|}{\left|\Delta_{1} \Delta_{2}\right|} \frac{\|m\|}{\Gamma(q-1)}\left[\sum_{i=1}^{m-2}\left|\delta_{i}\right|\left(\frac{1-\gamma}{q-\gamma-1}\right)^{1-\gamma}\right. \\
& \left.+\sum_{j=1}^{p-2}\left|\gamma_{j}\right|\left(\frac{1-\gamma}{q-\gamma-1}\right)^{1-\gamma} \frac{\eta^{q-\gamma}-\xi^{q-\gamma}}{q-\gamma}+|d|\left(\frac{1-\gamma}{q-\gamma-1}\right)^{1-\gamma}\right] \\
& +\max _{t \in[0,1]} \frac{\left|\Delta_{2} t-\Delta_{3}\right|}{\left|\Delta_{1} \Delta_{2}\right|}\left[\sum_{i=1}^{m-2}\left|\delta_{i}\right| \sigma_{i}^{q-1}+\sum_{j=1}^{p-2} \frac{\left|\gamma_{j}\right|}{q}\left|\eta_{j}^{q}-\xi_{j}^{q}\right|+|d|\right] \frac{d_{1} r^{\rho_{1}}+d_{2} r^{\rho_{2}}}{\Gamma(q)} \\
& +\frac{1}{\left|\Delta_{2}\right|} \frac{\|m\|}{\Gamma(q)}\left[\sum_{i=1}^{m-2}\left|\alpha_{i}\right|\left(\frac{1-\gamma}{q-\gamma}\right)^{1-\gamma}+\sum_{j=1}^{p-2}\left|r_{j}\right|\left(\frac{1-\gamma}{q-\gamma}\right)^{1-\gamma} \frac{\eta^{q-\gamma+1}-\xi^{q-\gamma+1}}{q-\gamma+1}\right. \\
& \left.+|b|\left(\frac{1-\gamma}{q-\gamma}\right)^{1-\gamma}\right] \\
& +\frac{1}{\left|\Delta_{2}\right|}\left[\sum_{i=1}^{m-2}\left|\alpha_{i}\right| \sigma_{i}^{q}+\sum_{j=1}^{p-2} \frac{\left|r_{j}\right|}{q+1}\left|\eta_{j}^{q+1}-\xi_{j}^{q+1}\right|+|b| \frac{d_{1} r^{\rho_{1}}+d_{2} r^{\rho_{2}}}{\Gamma(q+1)}\right. \\
&
\end{aligned}
$$

and

$$
\begin{aligned}
\left|\left({ }^{c} D^{\beta} F x\right)(t)\right| & \leq \int_{0}^{t} \frac{(t-s)^{q-\beta-1}}{\Gamma(q-\beta)}\left[m(s)+d_{1}|x(s)|^{\rho_{1}}+d_{2}\left|{ }^{c} D^{\beta} x(s)\right|^{\rho_{2}}\right] d s \\
& +\frac{1}{\left|\Delta_{1}\right|} \frac{t^{1-\beta}}{\Gamma(2-\beta)}\left[\sum_{i=1}^{m-2}\left|\delta_{i}\right| \int_{0}^{\sigma_{i}} \frac{\left(\sigma_{i}-s\right)^{q-2}}{\Gamma(q-1)}\left[m(s)+d_{1}|x(s)|^{\rho_{1}}+\left.\left.d_{2}\right|^{c} D^{\beta} x(s)\right|^{\rho_{2}}\right] d s\right. \\
& +\sum_{j=1}^{p-2}\left|\gamma_{j}\right| \int_{\xi_{j}}^{\eta_{j}}\left(\int_{0}^{s} \frac{(s-u)^{q-2}}{\Gamma(q-1)}\left[m(u)+d_{1}|x(u)|^{\rho_{1}}+d_{2}\left|{ }^{c} D^{\beta} x(u)\right|^{\rho_{2}}\right] d u\right) d s \\
& \left.+|d| \int_{0}^{1} \frac{(1-s)^{q-2}}{\Gamma(q-1)}\left[m(s)+d_{1}|x(s)|^{\rho_{1}}+d_{2}\left|{ }^{c} D^{\beta} x(s)\right|^{\rho_{2}}\right] d s\right] \\
\leq & \frac{\|m\|}{\Gamma(q-\beta)}\left(\frac{1-\gamma}{q-\beta-\gamma}\right)^{1-\gamma}+\frac{d_{1} r^{\rho_{1}}+d_{2} r^{\rho_{2}}}{\Gamma(q-\beta+1)} \\
& +\frac{\|m\|}{\left|\Delta_{1}\right|} \frac{1}{\Gamma(2-\beta)}\left(\frac{1-\gamma}{q-\gamma-1}\right)^{1-\gamma}\left[\sum_{i=1}^{m-2}\left|\delta_{i}\right|+\sum_{j=1}^{p-2}\left|\gamma_{j}\right| \frac{\eta^{q-\gamma}-\xi^{q-\gamma}}{q-\gamma}+|d|\right] \\
& +\frac{1}{\left|\Delta_{1}\right|} \frac{1}{\Gamma(2-\beta)}\left[\sum_{i=1}^{m-2}\left|\delta_{i}\right|+\sum_{j=1}^{p-2}\left|\gamma_{j}\right| \frac{\eta^{q-\gamma}-\xi^{q-\gamma}}{q-\gamma}+|d|\right] \frac{d_{1} r^{\rho_{1}}+d_{2} r^{\rho_{2}}}{\Gamma(q-\beta+1)} .
\end{aligned}
$$

From the above inequalities, we obtain

$$
\|F x\|_{X} \leq L+M\left[d_{1} r^{\rho_{1}}+d_{2} r^{\rho_{2}}\right]
$$


where

$$
\begin{aligned}
L= & \frac{\|m\|}{\Gamma(q)}\left(\frac{1-\gamma}{q-\gamma}\right)^{1-\gamma}+\max _{t \in[0,1]} \frac{\left|\Delta_{2} t-\Delta_{3}\right|}{\left|\Delta_{1} \Delta_{2}\right|} \frac{\|m\|}{\Gamma(q-1)}\left[\sum_{i=1}^{m-2}\left|\delta_{i}\right|\left(\frac{1-\gamma}{q-\gamma-1}\right)^{1-\gamma}\right. \\
& \left.+\sum_{j=1}^{p-2}\left|\gamma_{j}\right|\left(\frac{1-\gamma}{q-\gamma-1}\right)^{1-\gamma} \frac{\eta^{q-\gamma}-\xi^{q-\gamma}}{q-\gamma}+|d|\left(\frac{1-\gamma}{q-\gamma-1}\right)^{1-\gamma}\right] \\
& +\frac{1}{\left|\Delta_{2}\right|} \frac{\|m\|}{\Gamma(q)}\left[\sum_{i=1}^{m-2}\left|\alpha_{i}\right|\left(\frac{1-\gamma}{q-\gamma}\right)^{1-\gamma}+\sum_{j=1}^{p-2}\left|r_{j}\right|\left(\frac{1-\gamma}{q-\gamma}\right)^{1-\gamma} \frac{\eta^{q-\gamma+1}-\xi^{q-\gamma+1}}{q-\gamma+1}\right. \\
& \left.+|b|\left(\frac{1-\gamma}{q-\gamma}\right)^{1-\gamma}\right]+\frac{\|m\|}{\Gamma(q-\beta)}\left(\frac{1-\gamma}{q-\beta-\gamma}\right)^{1-\gamma} \\
& +\frac{\|m\|}{\left|\Delta_{1}\right|} \frac{1}{\Gamma(2-\beta)}\left(\frac{1-\gamma}{q-\gamma-1}\right)^{1-\gamma}\left[\sum_{i=1}^{m-2}\left|\delta_{i}\right|+\sum_{j=1}^{p-2}\left|\gamma_{j}\right| \frac{\eta^{q-\gamma}-\xi^{q-\gamma}}{q-\gamma}+|d|\right]
\end{aligned}
$$

and

$$
\begin{aligned}
M= & \frac{1}{\Gamma(q+1)}+\max _{t \in[0,1]} \frac{\left|\Delta_{2} t-\Delta_{3}\right|}{\left|\Delta_{1} \Delta_{2}\right|}\left[\sum_{i=1}^{m-2}\left|\delta_{i}\right| \sigma_{i}^{q-1}+\sum_{j=1}^{p-2} \frac{\left|\gamma_{j}\right|}{q}\left|\eta_{j}^{q}-\xi_{j}^{q}\right|+|d|\right] \frac{1}{\Gamma(q)} \\
& +\frac{1}{\left|\Delta_{2}\right|}\left[\sum_{i=1}^{m-2}\left|\alpha_{i}\right| \sigma_{i}^{q}+\sum_{j=1}^{p-2} \frac{\left|r_{j}\right|}{q+1}\left|\eta_{j}^{q+1}-\xi_{j}^{q+1}\right|+|b|\right] \frac{1}{\Gamma(q+1)}+\frac{1}{\Gamma(q-\beta+1)} \\
& +\frac{1}{\left|\Delta_{1}\right|} \frac{1}{\Gamma(2-\beta)}\left[\sum_{i=1}^{m-2}\left|\delta_{i}\right|+\sum_{j=1}^{p-2}\left|\gamma_{j}\right| \frac{\eta^{q-\gamma}-\xi^{q-\gamma}}{q-\gamma}+|d|\right] \frac{1}{\Gamma(q-\beta+1)} .
\end{aligned}
$$

Let $r$ be a positive number such that

$$
r \geq \max \left\{3 L,\left(3 M d_{1}\right)^{\frac{1}{1-\rho_{1}}},\left(3 M d_{2}\right)^{\frac{1}{1-\rho_{2}}}\right\} .
$$

Then, for any $x \in B_{r}$, it follows that

$$
\|F x\|_{X} \leq L+M\left[d_{1} r^{\rho_{1}}+d_{2} r^{\rho_{2}}\right] \leq \frac{r}{3}+\frac{r}{3}+\frac{r}{3}=r .
$$

In view of the continuity of $f$, it is easy to verify that $F$ is continuous.

Next, for every bounded subset $\bar{B}$ of $X$, we show that the families $F(\bar{B})$ and ${ }^{c} D^{\beta} F(\bar{B})$ are equicontinuous, where $\bar{B}$ is any bounded subset of $X$. Since $f$ is continuous, we can assume that $\left|f\left(t, x(t),{ }^{c} D^{\beta} x(t)\right)\right| \leq N$ for any $x \in \bar{B}$ and $t \in[0,1]$.

Now, for $0 \leq t_{1}<t_{2} \leq 1$, we have

$$
\begin{aligned}
& \left|(F x)\left(t_{2}\right)-(F x)\left(t_{1}\right)\right| \\
& \leq \mid \frac{1}{\Gamma(\alpha)} \int_{0}^{t_{1}}\left[\left(t_{2}-s\right)^{\alpha-1}-\left(t_{1}-s\right)^{\alpha-1}\right] f\left(s, x(s),{ }^{c} D^{\beta} x(s)\right) d s \\
& \quad+\frac{1}{\Gamma(\alpha)} \int_{t_{1}}^{t_{2}}\left(t_{2}-s\right)^{\alpha-1} f\left(s, x(s),{ }^{c} D^{\beta} x(t)\right) d s \mid
\end{aligned}
$$




$$
\begin{aligned}
& +\frac{\left|t_{2}-t_{1}\right|}{\left|\Delta_{1}\right|}\left[\sum_{i=1}^{m-2}\left|\delta_{i}\right| \int_{0}^{\sigma_{i}} \frac{\left(\sigma_{i}-s\right)^{q-2}}{\Gamma(q-1)} f\left(s, x(s),{ }^{c} D^{\beta} x(s)\right) d s\right. \\
& +\sum_{j=1}^{p-2}\left|\gamma_{j}\right| \int_{\xi_{j}}^{\eta_{j}}\left(\int_{0}^{s} \frac{(s-u)^{q-2}}{\Gamma(q-1)} f\left(u, x(u),{ }^{c} D^{\beta} x(u)\right) d u\right) d s \\
& \left.+|d| \int_{0}^{1} \frac{(1-s)^{q-2}}{\Gamma(q-1)} f\left(s, x(s),{ }^{c} D^{\beta} x(s)\right) d s\right] \\
& \leq \frac{N}{\Gamma(\alpha+1)}\left|2\left(t_{2}-t_{1}\right)^{\alpha}+t_{1}^{\alpha}-t_{2}^{\alpha}\right| \\
& +\frac{N\left|t_{2}-t_{1}\right|}{\left|\Delta_{1}\right|}\left[\sum_{i=1}^{m-2}\left|\delta_{i}\right| \frac{\sigma_{i}^{q-1}}{\Gamma(q)}+\sum_{j=1}^{p-2} \frac{\left|\gamma_{j}\right|}{\Gamma(q+1)}\left|\eta_{j}^{q}-\xi_{j}^{q}\right|+\frac{|d|}{\Gamma(q)}\right]
\end{aligned}
$$

and

$$
\begin{aligned}
& \left|\left({ }^{c} D^{\beta} F x\right)\left(t_{2}\right)-\left({ }^{c} D^{\beta} F x\right)\left(t_{1}\right)\right| \\
& \leq \frac{N}{\Gamma(q-\beta+1)}\left|2\left(t_{2}-t_{1}\right)^{q-\beta}+t_{1}^{q-\beta}-t_{2}^{q-\beta}\right| \\
& \quad+\frac{N}{\left|\Delta_{1}\right|} \frac{\left|t_{2}^{1-\beta}-t_{1}^{1-\beta}\right|}{\Gamma(2-\beta)}\left[\sum_{i=1}^{m-2}\left|\delta_{i}\right| \frac{\sigma_{i}^{q-1}}{\Gamma(q)}+\sum_{j=1}^{p-2} \frac{\left|\gamma_{j}\right|}{\Gamma(q+1)}\left|\eta_{j}^{q}-\xi_{j}^{q}\right|+\frac{|d|}{\Gamma(q)}\right] .
\end{aligned}
$$

In consequence, we obtain

$$
\sup _{x \in \bar{B}}\left|(F x)\left(t_{2}\right)-(F x)\left(t_{1}\right)\right|+\sup _{x \in \bar{B}}\left|\left({ }^{c} D^{\beta} F x\right)\left(t_{2}\right)-\left({ }^{c} D^{\beta} F x\right)\left(t_{1}\right)\right| \rightarrow 0 \quad \text { as } t_{2} \rightarrow t_{1},
$$

independent of $x \in \bar{B}$. Therefore the operator $F: B_{r} \rightarrow B_{r}$ is equicontinuous and uniformly bounded. Hence, by the Arzelá-Ascoli theorem, it follows that $F\left(B_{r}\right)$ is relatively compact in $X$. Therefore, the conclusion of Theorem 3.1 applies, and consequently problem (1.1)(1.2) has at least one solution on $[0,1]$. The proof is completed.

Corollary 3.3 Let $f:[0,1] \times \mathbb{R} \times \mathbb{R} \rightarrow \mathbb{R}$ be a continuous function such that $|f(t, x, y)| \leq$ $v(t), \forall(t, x, y) \in[0,1] \times \mathbb{R} \times \mathbb{R}$, where $v \in C\left([0,1], \mathbb{R}^{+}\right)$. Then the boundary value problem (1.1)-(1.2) has at least one solution on $[0,1]$.

For $\rho_{1}=\rho_{2}=1$, Theorem 3.2 takes the following form.

Corollary 3.4 Assume that $f:[0,1] \times \mathbb{R} \times \mathbb{R} \rightarrow \mathbb{R}$ is a continuous function satisfying the assumption: $|f(t, x, y)| \leq v(t)+d_{1}|x|+d_{2}|y|, \forall(t, x, y) \in[0,1] \times \mathbb{R} \times \mathbb{R}$, and $v \in L^{\frac{1}{\gamma}}\left([0,1], \mathbb{R}^{+}\right)$, $\gamma \in(0, q-1), d_{i} \geq 0, i=1,2$. Then problem (1.1)-(1.2) has at least one solution on $[0,1]$.

In our next result, we make use of Leray-Schauder's nonlinear alternative to prove the existence of solutions for problem (1.1)-(1.2).

Theorem 3.5 (Nonlinear alternative for single-valued maps [25]). Let $\mathcal{E}_{1}$ be a closed, convex subset of the Banach space $\mathcal{E}$ and $\mathcal{U}$ be an open subset of $\mathcal{E}_{1}$ with $0 \in \mathcal{U}$. Suppose that $\mathcal{F}: \overline{\mathcal{U}} \rightarrow \mathcal{E}_{1}$ is a continuous, compact map, that is, $\mathcal{F}\left(\overline{\mathcal{E}}_{1}\right)$ is a relatively compact subset of 
$\mathcal{E}_{1}$. Then either $\mathcal{F}$ has a fixed point in $\overline{\mathcal{E}}_{1}$ or there are $u \in \partial \mathcal{U}$ (the boundary of $\mathcal{U}$ in $\mathcal{E}_{1}$ ) and $\lambda \in(0,1)$ such that $u=\lambda \mathcal{F}(u)$.

Theorem 3.6 Let $f:[0,1] \times \mathbb{R} \times \mathbb{R} \rightarrow \mathbb{R}$ be a continuous function. Assume that:

$\left(A_{2}\right)$ There exist a function $m \in L^{\frac{1}{\gamma}}\left([0,1], \mathbb{R}^{+}\right), \gamma \in(0, q-1)$, and $\psi_{i}: \mathbb{R}^{+} \rightarrow \mathbb{R}^{+}, i=1,2$, is nondecreasing such that $|f(t, x, y)| \leq m(t)\left(\psi_{1}(|x|)+\psi_{2}(|y|)\right), \forall(t, x, y) \in[0,1] \times \mathbb{R} \times \mathbb{R}$;

$\left(A_{3}\right)$ There exists a constant $M>0$ such that

$$
\frac{M}{\left[\psi_{1}(M)+\psi_{2}(M)\right]\|m\|\left(Z_{1}+Z_{2}\right)}>1
$$

where $\|m\|=\left(\int_{0}^{1}|m(s)|^{\frac{1}{\gamma}} d s\right)^{\gamma}$ and $Z_{1}, Z_{2}$ are defined by (2.14) and (2.15), respectively. Then problem (1.1)-(1.2) has at least one solution on $[0,1]$.

Proof Firstly, we show that the operator $F: X \rightarrow X$ defined by (2.9) maps bounded sets into bounded sets in the space $X$. Let $B_{r}=\left\{x \in X:\|x\|_{X} \leq r\right\}, r>0$. For any $x \in B_{r}$, we have

$$
\begin{aligned}
& |(F x)(t)| \\
& \leq \int_{0}^{t} \frac{(t-s)^{q-1}}{\Gamma(q)} m(s)\left[\psi_{1}(|x(s)|)+\psi_{2}\left(\left|{ }^{c} D^{\beta} x(s)\right|\right)\right] d s \\
& +\max _{t \in[0,1]} \frac{\left|\Delta_{2} t-\Delta_{3}\right|}{\left|\Delta_{1} \Delta_{2}\right|}\left[\sum_{i=1}^{m-2}\left|\delta_{i}\right| \int_{0}^{\sigma_{i}} \frac{\left(\sigma_{i}-s\right)^{q-2}}{\Gamma(q-1)} m(s)\left[\psi_{1}(|x(s)|)+\psi_{2}\left(\left|{ }^{c} D^{\beta} x(s)\right|\right)\right] d s\right. \\
& +\sum_{j=1}^{p-2}\left|\gamma_{j}\right| \int_{\xi_{j}}^{\eta_{j}}\left(\int_{0}^{s} \frac{(s-u)^{q-2}}{\Gamma(q-1)} m(u)\left[\psi_{1}(|x(u)|)+\psi_{2}\left(\left|{ }^{c} D^{\beta} x(u)\right|\right)\right] d u\right) d s \\
& \left.+|d| \int_{0}^{1} \frac{(1-s)^{q-2}}{\Gamma(q-1)} m(s)\left[\psi_{1}(|x(s)|)+\psi_{2}\left(\left|{ }^{c} D^{\beta} x(s)\right|\right)\right] d s\right] \\
& +\frac{1}{\left|\Delta_{2}\right|}\left[\sum_{i=1}^{m-2}\left|\alpha_{i}\right| \int_{0}^{\sigma_{i}} \frac{\left(\sigma_{i}-s\right)^{q-1}}{\Gamma(q)} m(s)\left[\psi_{1}(|x(s)|)+\psi_{2}\left(\left|{ }^{c} D^{\beta} x(s)\right|\right)\right] d s\right. \\
& +\sum_{j=1}^{p-2}\left|r_{j}\right| \int_{\xi_{j}}^{\eta_{j}}\left(\int_{0}^{s} \frac{(s-u)^{q-1}}{\Gamma(q)} m(u)\left[\psi_{1}(|x(u)|)+\psi_{2}\left(\left|{ }^{c} D^{\beta} x(u)\right|\right)\right]\right) d s \\
& \left.+|b| \int_{0}^{1} \frac{(1-s)^{q-1}}{\Gamma(q)} m(s)\left[\psi_{1}(|x(s)|)+\psi_{2}\left(\left|{ }^{c} D^{\beta} x(s)\right|\right)\right] d s\right] \\
& \leq\left\{\frac{\|m\|}{\Gamma(q)}\left(\frac{1-\gamma}{q-\gamma}\right)^{1-\gamma}+\max _{t \in[0,1]} \frac{\left|\Delta_{2} t-\Delta_{3}\right|}{\left|\Delta_{1} \Delta_{2}\right|} \frac{\|m\|}{\Gamma(q-1)}\left[\sum_{i=1}^{m-2}\left|\delta_{i}\right|\left(\frac{1-\gamma}{q-\gamma-1}\right)^{1-\gamma}\right.\right. \\
& \left.+\sum_{j=1}^{p-2}\left|\gamma_{j}\right|\left(\frac{1-\gamma}{q-\gamma-1}\right)^{1-\gamma} \frac{\eta^{q-\gamma}-\xi^{q-\gamma}}{q-\gamma}+|d|\left(\frac{1-\gamma}{q-\gamma-1}\right)^{1-\gamma}\right] \\
& +\frac{1}{\left|\Delta_{2}\right|} \frac{\|m\|}{\Gamma(q)}\left[\sum_{i=1}^{m-2}\left|\alpha_{i}\right|\left(\frac{1-\gamma}{q-\gamma}\right)^{1-\gamma}+\sum_{j=1}^{p-2}\left|r_{j}\right|\left(\frac{1-\gamma}{q-\gamma}\right)^{1-\gamma} \frac{\eta^{q-\gamma+1}-\xi^{q-\gamma+1}}{q-\gamma+1}\right. \\
& \left.\left.+|b|\left(\frac{1-\gamma}{q-\gamma}\right)^{1-\gamma}\right]\right\}\left[\psi_{1}(r)+\psi_{2}(r)\right]
\end{aligned}
$$


and

$$
\begin{aligned}
\left|\left({ }^{c} D^{\beta} F x\right)(t)\right| & \leq \int_{0}^{t} \frac{(t-s)^{q-\beta-1}}{\Gamma(q-\beta)} m(s)\left[\psi_{1}(|x(s)|)+\psi_{2}\left(\left|{ }^{c} D^{\beta} x(s)\right|\right)\right] d s \\
& +\frac{1}{\left|\Delta_{1}\right|} \frac{t^{1-\beta}}{\Gamma(2-\beta)}\left[\sum_{i=1}^{m-2}\left|\delta_{i}\right| \int_{0}^{\sigma_{i}} \frac{\left(\sigma_{i}-s\right)^{q-2}}{\Gamma(q-1)} m(s)\left[\psi_{1}(|x(s)|)+\psi_{2}\left(\left|{ }^{c} D^{\beta} x(s)\right|\right)\right] d s\right. \\
& +\sum_{j=1}^{p-2}\left|\gamma_{j}\right| \int_{\xi_{j}}^{\eta_{j}}\left(\int_{0}^{s} \frac{(s-u)^{q-2}}{\Gamma(q-1)} m(u)\left[\psi_{1}(|x(u)|)+\psi_{2}\left(\left|{ }^{c} D^{\beta} x(u)\right|\right)\right] d u\right) d s \\
& \left.+|d| \int_{0}^{1} \frac{(1-s)^{q-2}}{\Gamma(q-1)} m(s)\left[\psi_{1}(|x(s)|)+\psi_{2}\left(\left|{ }^{c} D^{\beta} x(s)\right|\right)\right] d s\right] \\
\leq & \left.\left\{\frac{\|m\|}{\Gamma(q-\beta)}\left(\frac{1-\gamma}{q-\beta-\gamma}\right)^{1-\gamma}+\frac{\|m\|}{\left|\Delta_{1}\right|} \frac{1}{\Gamma(2-\beta)}\right]\right\}\left[\psi_{1}(r)+\psi_{2}(r)\right] .
\end{aligned}
$$

From the above inequalities, we find that

$$
\|F x\|_{X} \leq\left[\psi_{1}(r)+\psi_{2}(r)\right]\|m\|\left(Z_{1}+Z_{2}\right) .
$$

As established in the proof of Theorem 3.2, one can show that $F$ is equicontinuous on bounded sets of $X$. Finally, for $\lambda \in(0,1)$, let $x=\lambda F x$. Then it is easy to obtain that

$$
\frac{\|x\|_{X}}{\left[\psi_{1}\left(\|x\|_{X}\right)+\psi_{2}\left(\|x\|_{X}\right)\right]\|m\|\left(Z_{1}+Z_{2}\right)} \leq 1 .
$$

In view of $\left(A_{3}\right)$, there exists $M$ such that $\|x\| \neq M$. Let us set

$$
V=\left\{x \in C([0,1], \mathbb{R}):\|x\|_{X}<M\right\}
$$

Note that the operator $F: \bar{V} \rightarrow C([0,1], \mathbb{R})$ is continuous and completely continuous. From the choice of $V$, there is no $x \in \partial V$ such that $x=\lambda F x$ for some $\lambda \in(0,1)$. Consequently, by the nonlinear alternative of Leray-Schauder type (Theorem 3.5), we deduce that $F$ has a fixed point $x \in \bar{V}$, which is a solution of problem (1.1)-1.2). This completes the proof.

Our last existence result is based on Krasnoselskii's fixed point theorem.

Lemma 3.7 (Krasnoselskii's fixed point theorem [26]) Let $Y$ be a closed, bounded, convex, and nonempty subset of a Banach space $X$. Let $A, B$ be the operators such that (i) $A y_{1}+B y_{2} \in$ $Y$ whenever $y_{1}, y_{2} \in Y$; (ii) $A$ is compact and continuous; (iii) $B$ is a contraction mapping. Then there exists $y_{3} \in Y$ such that $y_{3}=A y_{3}+B y_{3}$.

Theorem 3.8 Let $f:[0,1] \times \mathbb{R} \times \mathbb{R} \rightarrow \mathbb{R}$ be a continuous function satisfying the following conditions: 
$\left(A_{4}\right)\left|f\left(t, x_{1}, y_{1}\right)-f\left(t, x_{2}, y_{2}\right)\right| \leq m(t)\left(\left|x_{1}-x_{2}\right|+\left|y_{1}-y_{2}\right|\right)$ for $t \in[0,1], x_{i}, y_{i} \in \mathbb{R}, i=1,2$ and $\gamma \in(0, q-1)$ and the function $m:[0,1] \rightarrow \mathbb{R}^{+}$is Lebesgue integrable with power $\frac{1}{\gamma}$, that is,

$$
m \in L^{\frac{1}{\gamma}}\left([0,1], \mathbb{R}^{+}\right) \quad \text { with }\|m\|=\left(\int_{0}^{1}|m(s)|^{\frac{1}{\gamma}} d s\right)^{\gamma}
$$

$\left(A_{5}\right)|f(t, x, y)| \leq m(t), \forall(t, x, y) \in[0,1] \times \mathbb{R} \times \mathbb{R}$, and $m \in L^{\frac{1}{\gamma}}\left([0,1], \mathbb{R}^{+}\right), \gamma \in(0, q-1)$.

Then problem (1.1)-(1.2) has at least one solution on $[0,1]$ provided that

$$
\|m\|\left(Z_{1}-\frac{1}{\Gamma(q)}\left(\frac{1-\gamma}{q-\gamma}\right)^{1-\gamma}+Z_{2}-\frac{1}{\Gamma(q-\beta)}\left(\frac{1-\gamma}{q-\beta-\gamma}\right)^{1-\gamma}\right)<1
$$

where $Z_{1}, Z_{2}$ are defined by (2.14) and (2.15), respectively.

Proof Selecting $\rho>L$, we define $B_{\rho}=\left\{x \in \mathcal{C}:\|x\|_{X} \leq \rho\right\}$ and introduce the operators $A$ and $B$ on $B_{\rho}$ as follows:

$$
A(x)(t)=\int_{0}^{t} \frac{(t-s)^{q-1}}{\Gamma(q)} f\left(s, x(s),{ }^{c} D^{\beta} x(s)\right) d s
$$

and

$$
\begin{aligned}
B(x)(t)= & \frac{\left(\Delta_{2} t-\Delta_{3}\right)}{\Delta_{1} \Delta_{2}}\left[\sum_{i=1}^{m-2} \delta_{i} \int_{0}^{\sigma_{i}} \frac{\left(\sigma_{i}-s\right)^{q-2}}{\Gamma(q-1)} f\left(s, x(s),{ }^{c} D^{\beta} x(s)\right) d s\right. \\
& +\sum_{j=1}^{p-2} \gamma_{j} \int_{\xi_{j}}^{\eta_{j}}\left(\int_{0}^{s} \frac{(s-u)^{q-2}}{\Gamma(q-1)} f\left(u, x(u),{ }^{c} D^{\beta} x(u)\right) d u\right) d s \\
& \left.-d \int_{0}^{1} \frac{(1-s)^{q-2}}{\Gamma(q-1)} f\left(s, x(s),{ }^{c} D^{\beta} x(s)\right) d s\right] \\
& +\frac{1}{\Delta_{2}}\left[\sum_{i=1}^{m-2} \alpha_{i} \int_{0}^{\sigma_{i}} \frac{\left(\sigma_{i}-s\right)^{q-1}}{\Gamma(q)} f\left(s, x(s),{ }^{c} D^{\beta} x(s)\right) d s\right. \\
& +\sum_{j=1}^{p-2} r_{j} \int_{\xi_{j}}^{\eta_{j}}\left(\int_{0}^{s} \frac{(s-u)^{q-1}}{\Gamma(q)} f\left(u, x(u),{ }^{c} D^{\beta} x(u)\right) d u\right) d s \\
& \left.-b \int_{0}^{1} \frac{(1-s)^{q-1}}{\Gamma(q)} f\left(s, x(s),{ }^{c} D^{\beta} x(s)\right) d s\right] .
\end{aligned}
$$

For any $x, y \in B_{\rho}$, as in the proof of Theorem 3.2, it can be shown that $\|A x+B x\|_{X} \leq L<\rho$. This shows that $A x+B y \in B_{\rho}$. The operator $A$ is completely continuous as in Theorem (3.2). Using assumption $\left(A_{5}\right)$, as in the proof of Theorem 4.5 , it can be shown that the operator $B$ is a contraction with the aid of (3.1). Thus all the assumptions of Lemma 3.7 are satisfied. Hence the conclusion of Lemma 3.7 implies that problem (1.1)-(1.2) has at least one solution on $[0,1]$. 


\section{Uniqueness results}

In this section we discuss the uniqueness of solutions for problem (1.1)-(1.2) by using a variety of fixed point theorems.

Our first result relies on Boyd and Wong's fixed point theorem. For a quick reference, we recall it here.

Definition 4.1 Let $E$ be a real Banach space. A nonempty closed set $P \subset E$ is said to be a cone provided that (i) $a u+b v \in P$ for all $u, v \in P$ and all $a, b \geq 0$, and (ii) $u,-u \in P$ implies that $u=0$.

Definition 4.2 A mapping $F$ acting in a Banach space $E$ is said to be a nonlinear contraction if there exists a continuous nondecreasing function $\Psi: \mathbb{R}^{+} \rightarrow \mathbb{R}^{+}\left(\mathbb{R}^{+}\right.$denotes the set of nonnegative real numbers) such that $\Psi(0)=0, \Psi(\xi)<\xi$ for all $\xi>0$ and that $\|F x-F y\| \leq \Psi(\|x-y\|), \forall x, y \in E$.

Lemma 4.3 (Boyd and Wong) [27]. Let E be a Banach space, and let $F: E \rightarrow E$ be a nonlinear contraction. Then $F$ has a unique fixed point in $E$.

Theorem 4.4 Assume that $f:[0,1] \times \mathbb{R} \times \mathbb{R} \rightarrow \mathbb{R}$ is a continuous function satisfying the condition

$$
\begin{aligned}
\left|f\left(t, x_{1}, y_{1}\right)-f\left(t, x_{2}, y_{2}\right)\right| \leq & h_{1}(t) \frac{\left|x_{1}-x_{2}\right|}{H^{*}+\left|x_{1}-x_{2}\right|} \\
& +h_{2}(t) \frac{\left|y_{1}-y_{2}\right|}{H^{*}+\left|y_{1}-y_{2}\right|}, \quad t \in(0,1),
\end{aligned}
$$

$x_{i}, y_{i} \in \mathbb{R}, i=1,2$, where $h_{1}, h_{2}:(0,1) \rightarrow \mathbb{R}^{+}$are continuous functions with

$$
H^{*}=\frac{1}{\Omega+\Omega_{1}}\left(\left\|h_{1}\right\|+\left\|h_{2}\right\|\right),
$$

and $\Omega, \Omega_{1}$ are respectively defined by (2.12) and (2.13). Then the boundary value problem (1.1)-(1.2) has a unique solution.

Proof Consider the operator $F: X \rightarrow X$ defined by (2.9). Let the continuous nondecreasing function $\Psi: \mathbb{R}^{+} \rightarrow \mathbb{R}^{+}$be defined by

$$
\Psi(\xi)=\frac{H^{*} \xi}{H^{*}+\xi}, \quad \forall \xi \geq 0
$$

Observe that $\Psi(0)=0$ and $\Psi(\xi)<\xi$ for all $\xi>0$.

For $x, y \in X$, we can find that

$$
\begin{aligned}
& \left|f\left(s, x(s),{ }^{c} D^{\beta} x(s)\right)-f\left(s, y(s),{ }^{c} D^{\beta} y(s)\right)\right| \\
& \quad \leq h_{1}(s) \frac{|x(s)-y(s)|}{H^{*}+|x(s)-y(s)|}+h_{2}(s) \frac{\left|{ }^{c} D^{\beta} x(s)-{ }^{c} D^{\beta} y(s)\right|}{H^{*}+\left|{ }^{c} D^{\beta} x(s)-{ }^{c} D^{\beta} y(s)\right|} \\
& \quad \leq \frac{\left\|h_{1}\right\|+\left\|h_{2}\right\|}{H^{*}} \Psi\left(\|x-y\|_{X}\right) .
\end{aligned}
$$


Then

$$
\begin{aligned}
|(F x)(t)-(F y)(t)| & \mid \int_{0}^{t} \frac{(t-s)^{q-1}}{\Gamma(q)}\left[f\left(s, x(s), D^{c} D^{\beta} x(s)\right)-f\left(s, y(s),{ }^{c} D^{\beta} y(s)\right)\right] d s \\
& +\frac{\left(\Delta_{2} t-\Delta_{3}\right)}{\Delta_{1} \Delta_{2}}\left[\sum_{i=1}^{m-2} \delta_{i} \int_{0}^{\sigma_{i}} \frac{\left(\sigma_{i}-s\right)^{q-2}}{\Gamma(q-1)}\left[f\left(s, x(s),{ }^{c} D^{\beta} x(s)\right)-f\left(s, y(s),{ }^{c} D^{\beta} y(s)\right)\right] d s\right. \\
& +\sum_{j=1}^{p-2} \gamma_{j} \int_{\xi_{j}}^{\eta_{j}}\left(\int_{0}^{s} \frac{(s-u)^{q-2}}{\Gamma(q-1)}\left[f\left(u, x(u),{ }^{c} D^{\beta} x(u)\right)-f\left(u, y(u),{ }^{c} D^{\beta} y(u)\right)\right] d u\right) d s \\
& \left.-d \int_{0}^{1} \frac{(1-s)^{q-2}}{\Gamma(q-1)}\left[f\left(s, x(s),{ }^{c} D^{\beta} x(s)\right)-f\left(s, y(s),{ }^{c} D^{\beta} y(s)\right)\right] d s\right] \\
& +\frac{1}{\Delta_{2}}\left[\sum_{i=1}^{m-2} \alpha_{i} \int_{0}^{\sigma_{i}} \frac{\left(\sigma_{i}-s\right)^{q-1}}{\Gamma(q)}\left[f\left(s, x(s),{ }^{c} D^{\beta} x(s)\right)-f\left(s, y(s),{ }^{c} D^{\beta} y(s)\right)\right] d s\right. \\
& +\sum_{j=1}^{p-2} r_{j} \int_{\xi_{j}}^{\eta_{j}}\left(\int_{0}^{s} \frac{(s-u)^{q-1}}{\Gamma(q)}\left[f\left(u, x(u),{ }^{c} D^{\beta} x(u)\right)-f\left(u, y(u),{ }^{c} D^{\beta} y(u)\right)\right] d u\right) d s \\
& \left.-b \int_{0}^{1} \frac{(1-s)^{q-1}}{\Gamma(q)}\left[f\left(s, x(s),{ }^{c} D^{\beta} x(s)\right)-f\left(s, y(s),{ }^{c} D^{\beta} y(s)\right)\right] d s\right] \mid \\
\leq & \Omega\left(\left\|h_{1}\right\|+\left\|h_{2}\right\|\right) \frac{\|x-y\|_{X}}{H^{*}+\|x-y\|_{X}}
\end{aligned}
$$

and

$$
\begin{aligned}
& \left|\left({ }^{c} D^{\beta} F x\right)(t)-\left({ }^{c} D^{\beta} F y\right)(t)\right| \\
& =\mid \int_{0}^{t} \frac{(t-s)^{q-\beta-1}}{\Gamma(q-\beta)}\left[f\left(s, x(s),{ }^{c} D^{\beta} x(s)\right)-f\left(s, y(s),{ }^{c} D^{\beta} y(s)\right)\right] d s \\
& \quad+\frac{1}{\Delta_{1}} \frac{t^{1-\beta}}{\Gamma(2-\beta)}\left[\sum_{i=1}^{m-2} \delta_{i} \int_{0}^{\sigma_{i}} \frac{\left(\sigma_{i}-s\right)^{q-2}}{\Gamma(q-1)}\left[f\left(s, x(s),{ }^{c} D^{\beta} x(s)\right)-f\left(s, y(s),{ }^{c} D^{\beta} y(s)\right)\right] d s\right. \\
& \quad+\sum_{j=1}^{p-2} \gamma_{j} \int_{\xi_{j}}^{\eta_{j}}\left(\int_{0}^{s} \frac{(s-u)^{q-2}}{\Gamma(q-1)}\left[f\left(u, x(u),{ }^{c} D^{\beta} x(u)\right)-f\left(u, y(u),{ }^{c} D^{\beta} y(u)\right)\right] d u\right) d s \\
& \left.\quad-d \int_{0}^{1} \frac{(1-s){ }^{q-2}}{\Gamma(q-1)}\left[f\left(s, x(s),{ }^{c} D^{\beta} x(s)\right)-f\left(s, y(s),{ }^{c} D^{\beta} y(s)\right)\right] d s\right] \mid \\
& \leq \Omega_{1}\left(\left\|h_{1}\right\|+\left\|h_{2}\right\|\right) \frac{\|x-y\|_{X}}{H^{*}+\|x-y\|_{X}} .
\end{aligned}
$$

Then $\|F x-F y\|_{X} \leq \Psi\left(\|x-y\|_{X}\right)$ and $F$ is a nonlinear contraction and it has a unique fixed point in $X$ by Lemma 4.3. This completes the proof.

In the following result, we establish the uniqueness of solutions for problem (1.1)-(1.2) by applying Banach's fixed point theorem together with Hölder's inequality. 
Theorem 4.5 Let $f:[0,1] \times \mathbb{R} \times \mathbb{R} \rightarrow \mathbb{R}$ be a continuous function satisfying assumption $\left(A_{4}\right)$. Then there exists a unique solution for problem $(1.1)-(1.2)$ on $[0,1]$ if

$$
\|m\|\left(Z_{1}+Z_{2}\right)<1
$$

where $Z_{1}, Z_{2}$ are defined by (2.14) and (2.15), respectively.

Proof For $x, y \in X$ and for each $t \in[0,1]$, by Hölder's inequality, we have

$$
\begin{aligned}
& |(F x)(t)-(F y)(t)| \\
& \leq \mid \int_{0}^{t} \frac{(t-s)^{q-1}}{\Gamma(q)}\left[f\left(s, x(s),{ }^{c} D^{\beta} x(s)\right)-f\left(s, y(s),{ }^{c} D^{\beta} y(s)\right)\right] d s \\
& +\frac{\left(\Delta_{2} t-\Delta_{3}\right)}{\Delta_{1} \Delta_{2}}\left[\sum_{i=1}^{m-2} \delta_{i} \int_{0}^{\sigma_{i}} \frac{\left(\sigma_{i}-s\right)^{q-2}}{\Gamma(q-1)}\left[f\left(s, x(s),{ }^{c} D^{\beta} x(s)\right)-f\left(s, y(s),{ }^{c} D^{\beta} y(s)\right)\right] d s\right. \\
& +\sum_{j=1}^{p-2} \gamma_{j} \int_{\xi_{j}}^{\eta_{j}}\left(\int_{0}^{s} \frac{(s-u)^{q-2}}{\Gamma(q-1)}\left[f\left(u, x(u),{ }^{c} D^{\beta} x(u)\right)-f\left(u, y(u),{ }^{c} D^{\beta} y(u)\right)\right] d u\right) d s \\
& \left.-d \int_{0}^{1} \frac{(1-s)^{q-2}}{\Gamma(q-1)}\left[f\left(s, x(s),{ }^{c} D^{\beta} x(s)\right)-f\left(s, y(s),{ }^{c} D^{\beta} y(s)\right)\right] d s\right] \\
& +\frac{1}{\Delta_{2}}\left[\sum_{i=1}^{m-2} \alpha_{i} \int_{0}^{\sigma_{i}} \frac{\left(\sigma_{i}-s\right)^{q-1}}{\Gamma(q)}\left[f\left(s, x(s),{ }^{c} D^{\beta} x(s)\right)-f\left(s, y(s),{ }^{c} D^{\beta} y(s)\right)\right] d s\right. \\
& +\sum_{j=1}^{p-2} r_{j} \int_{\xi_{j}}^{\eta_{j}}\left(\int_{0}^{s} \frac{(s-u)^{q-1}}{\Gamma(q)}\left[f\left(u, x(u),{ }^{c} D^{\beta} x(u)\right)-f\left(u, y(u),{ }^{c} D^{\beta} y(u)\right)\right] d u\right) d s \\
& \left.-b \int_{0}^{1} \frac{(1-s)^{q-1}}{\Gamma(q)}\left[f\left(s, x(s),{ }^{c} D^{\beta} x(s)\right)-f\left(s, y(s),{ }^{c} D^{\beta} y(s)\right)\right] d s\right] \\
& \leq \int_{0}^{t} \frac{(t-s)^{q-1}}{\Gamma(q)} m(s)\left(|x(s)-y(s)|+\left|{ }^{c} D^{\beta} x(s)-{ }^{c} D^{\beta} y(s)\right|\right) d s \\
& +\max _{t \in[0,1]} \frac{\left|\Delta_{2} t-\Delta_{3}\right|}{\left|\Delta_{1} \Delta_{2}\right|}\left[\sum_{i=1}^{m-2}\left|\delta_{i}\right| \int_{0}^{\sigma_{i}} \frac{\left(\sigma_{i}-s\right)^{q-2}}{\Gamma(q-1)} m(s)(|x(s)-y(s)|\right. \\
& \left.+\left|{ }^{c} D^{\beta} x(s)-{ }^{c} D^{\beta} y(s)\right|\right) d s \\
& +\sum_{j=1}^{p-2}\left|\gamma_{j}\right| \int_{\xi_{j}}^{\eta_{j}}\left(\int_{0}^{s} \frac{(s-u)^{q-2}}{\Gamma(q-1)} m(u)\left(|x(u)-y(u)|+\left|{ }^{c} D^{\beta} x(u)-{ }^{c} D^{\beta} y(u)\right|\right) d u\right) d s \\
& \left.+|d| \int_{0}^{1} \frac{(1-s)^{q-2}}{\Gamma(q-1)} m(s)\left(|x(s)-y(s)|+\left|{ }^{c} D^{\beta} x(s)-{ }^{c} D^{\beta} y(s)\right|\right) d s\right] \\
& +\frac{1}{\left|\Delta_{2}\right|}\left[\sum_{i=1}^{m-2}\left|\alpha_{i}\right| \int_{0}^{\sigma_{i}} \frac{\left(\sigma_{i}-s\right)^{q-1}}{\Gamma(q)} m(s)\left(|x(s)-y(s)|+\left|{ }^{c} D^{\beta} x(s)-{ }^{c} D^{\beta} y(s)\right|\right) d s\right. \\
& +\sum_{j=1}^{p-2} r_{j} \int_{\xi_{j}}^{\eta_{j}}\left(\int_{0}^{s} \frac{(s-u)^{q-1}}{\Gamma(q)} m(u)\left(|x(u)-y(u)|+\left|{ }^{c} D^{\beta} x(u)-{ }^{c} D^{\beta} y(u)\right|\right) d u\right) d s
\end{aligned}
$$


Ahmad et al. Advances in Difference Equations ( 2018) 2018:89

Page 16 of 20

$$
\begin{aligned}
& \left.+|b| \int_{0}^{1} \frac{(1-s)^{q-1}}{\Gamma(q)} m(s)\left(|x(s)-y(s)|+\left|{ }^{c} D^{\beta} x(s)-{ }^{c} D^{\beta} y(s)\right|\right) d s\right] \\
& \leq \frac{\|x-y\|_{X}}{\Gamma(q)}\left(\int_{0}^{t}\left((t-s)^{q-1}\right)^{\frac{1}{1-\gamma}} d s\right)^{1-\gamma}\left(\int_{0}^{1}(m(s))^{1 / \gamma} d s\right)^{\gamma} \\
& +\max _{t \in[0,1]} \frac{\left|\Delta_{2} t-\Delta_{3}\right|}{\left|\Delta_{1} \Delta_{2}\right|} \frac{\|x-y\|_{X}}{\Gamma(q-1)}\left[\sum_{i=1}^{m-2}\left|\delta_{i}\right|\left(\int_{0}^{\sigma_{i}}\left(\left(\sigma_{i}-s\right)^{q-2}\right)^{\frac{1}{1-\gamma}} d s\right)^{1-\gamma}\right. \\
& \times\left(\int_{0}^{1}(m(s))^{1 / \gamma} d s\right)^{\gamma} \\
& +\sum_{j=1}^{p-2}\left|\gamma_{j}\right| \int_{\xi_{j}}^{\eta_{j}}\left[\left(\int_{0}^{s}\left((s-u)^{q-2}\right)^{\frac{1}{1-\gamma}} d u\right)^{1-\gamma}\left(\int_{0}^{1}(m(u))^{1 / \gamma} d u\right)^{\gamma}\right] d s \\
& \left.+|d|\left(\int_{0}^{1}\left((1-s)^{q-2}\right)^{\frac{1}{1-\gamma}} d s\right)^{1-\gamma}\left(\int_{0}^{1}(m(s))^{1 / \gamma} d s\right)^{\gamma}\right] \\
& +\frac{1}{\left|\Delta_{2}\right|} \frac{\|x-y\|_{X}}{\Gamma(q)}\left[\sum_{i=1}^{m-2}\left|\alpha_{i}\right|\left(\int_{0}^{\sigma_{i}}\left(\left(\sigma_{i}-s\right)^{q-1}\right)^{\frac{1}{1-\gamma}} d s\right)^{1-\gamma}\left(\int_{0}^{1}(m(s))^{1 / \gamma} d s\right)^{\gamma}\right. \\
& +\sum_{j=1}^{p-2}\left|r_{j}\right| \int_{\xi_{j}}^{\eta_{j}}\left[\left(\int_{0}^{s}\left((s-u)^{q-1}\right)^{\frac{1}{1-\gamma}} d u\right)^{1-\gamma}\left(\int_{0}^{1}(m(u))^{1 / \gamma} d u\right)^{\gamma}\right] d s \\
& \left.+|b|\left(\int_{0}^{1}\left((1-s)^{q-1}\right)^{\frac{1}{1-\gamma}} d s\right)^{1-\gamma}\left(\int_{0}^{1}(m(s))^{1 / \gamma} d s\right)^{\gamma}\right] \\
& \leq \frac{\|m\|\|x-y\|_{X}}{\Gamma(q)}\left(\frac{1-\gamma}{q-\gamma}\right)^{1-\gamma} \\
& +\max _{t \in[0,1]} \frac{\left|\Delta_{2} t-\Delta_{3}\right|}{\left|\Delta_{1} \Delta_{2}\right|} \frac{\|m\|\|x-y\|_{X}}{\Gamma(q-1)}\left[\sum_{i=1}^{m-2}\left|\delta_{i}\right|\left(\frac{1-\gamma}{q-\gamma-1}\right)^{1-\gamma}\right. \\
& \left.+\sum_{j=1}^{p-2}\left|\gamma_{j}\right|\left(\frac{1-\gamma}{q-\gamma-1}\right)^{1-\gamma} \frac{\eta^{q-\gamma}-\xi^{q-\gamma}}{q-\gamma}+|d|\left(\frac{1-\gamma}{q-\gamma-1}\right)^{1-\gamma}\right] \\
& +\frac{1}{\left|\Delta_{2}\right|} \frac{\|m\|\|x-y\|_{X}}{\Gamma(q)}\left[\sum_{i=1}^{m-2}\left|\alpha_{i}\right|\left(\frac{1-\gamma}{q-\gamma}\right)^{1-\gamma}+\sum_{j=1}^{p-2}\left|r_{j}\right|\left(\frac{1-\gamma}{q-\gamma}\right)^{1-\gamma} \frac{\eta^{q-\gamma+1}-\xi^{q-\gamma+1}}{q-\gamma+1}\right. \\
& \left.+|b|\left(\frac{1-\gamma}{q-\gamma}\right)^{1-\gamma}\right] \\
& =Z_{1}\|m\|\|x-y\|_{X} .
\end{aligned}
$$

Similarly, we have

$$
\begin{aligned}
& \left|\left({ }^{c} D^{\beta} F x\right)(t)-\left({ }^{c} D^{\beta} F y\right)(t)\right| \\
& \leq \int_{0}^{t} \frac{(t-s)^{q-\beta-1}}{\Gamma(q-\beta)} m(s)\left(|x(s)-y(s)|+\left|{ }^{c} D^{\beta} x(s)-{ }^{c} D^{\beta} y(s)\right|\right) d s \\
& \quad+\frac{1}{\left|\Delta_{1}\right|} \frac{t^{1-\beta}}{\Gamma(2-\beta)}\left[\sum_{i=1}^{m-2}\left|\delta_{i}\right| \int_{0}^{\sigma_{i}} \frac{\left(\sigma_{i}-s\right)^{q-2}}{\Gamma(q-1)}\right.
\end{aligned}
$$




$$
\begin{aligned}
& \times m(s)\left(|x(s)-y(s)|+\left|{ }^{c} D^{\beta} x(s)-{ }^{c} D^{\beta} y(s)\right|\right) d s \\
& +\sum_{j=1}^{p-2}\left|\gamma_{j}\right| \int_{\xi_{j}}^{\eta_{j}}\left(\int_{0}^{s} \frac{(s-u)^{q-2}}{\Gamma(q-1)} m(u)\left(|x(u)-y(u)|+\left|{ }^{c} D^{\beta} x(u)-{ }^{c} D^{\beta} y(u)\right|\right) d u\right) d s \\
& \left.+|d| \int_{0}^{1} \frac{(1-s)^{q-2}}{\Gamma(q-1)} m(s)\left(|x(s)-y(s)|+\left|{ }^{c} D^{\beta} x(s)-{ }^{c} D^{\beta} y(s)\right|\right) d s\right] \\
\leq & \left\{\frac{1}{\Gamma(q-\beta)}\left(\frac{1-\gamma}{q-\beta-\gamma}\right)^{1-\gamma}+\frac{1}{\left|\Delta_{1}\right|} \frac{1}{\Gamma(2-\beta)}\left(\frac{1-\gamma}{q-\gamma-1}\right)^{1-\gamma}\left[\sum_{i=1}^{m-2}\left|\delta_{i}\right|\right.\right. \\
& \left.\left.+\sum_{j=1}^{p-2}\left|\gamma_{j}\right| \frac{\eta_{j}^{q-\gamma}-\xi_{j}^{q-\gamma}}{q-\gamma}+|d|\right]\right\}\|m\|\|x-y\|_{X} \\
= & Z_{2}\|m\|\|x-y\|_{X} .
\end{aligned}
$$

From the above inequalities, we obtain

$$
\|F x-F y\|_{X} \leq\left(Z_{1}+Z_{2}\right)\|m\|\|x-y\|_{X}
$$

In view of the given condition $\|m\|\left(Z_{1}+Z_{2}\right)<1$, it follows that the mapping $F$ is a contraction. Hence, by the Banach fixed point theorem, $F$ has a unique fixed point which is a unique solution of problem (1.1)-(1.2). This completes the proof.

Corollary 4.6 Suppose that the continuous function $f:[0,1] \times \mathbb{R} \times \mathbb{R} \rightarrow \mathbb{R}$ satisfies the following assumption:

$\left(A_{4}\right)^{\prime}\left|f\left(t, x_{1}, y_{1}\right)-f\left(t, x_{2}, y_{2}\right)\right| \leq L\left(\left|x_{1}-x_{2}\right|+\left|y_{1}-y_{2}\right|\right)$ for $t \in[0,1], x_{i}, y_{i} \in \mathbb{R}, i=1,2$, and $L>0$ is a constant.

If $\Omega+\Omega_{1}<1$, where $\Omega, \Omega_{1}$ are defined by (2.12) and (2.13), respectively, then the boundary value problem (1.1)-(1.2) has a unique solution on $[0,1]$.

\section{Examples}

Let us consider problem (1.1)-(1.2) with specific data:

$$
\begin{aligned}
& q=3 / 2, \quad \beta=1 / 2, \quad \gamma=1 / 3 \in(0,1 / 2), \quad m=6, \quad p=5, \\
& a=b=c=d=1, \quad r_{1}=1 / 24, \quad r_{2}=1 / 12, \\
& r_{3}=1 / 6, \quad \alpha_{1}=1 / 25, \quad \alpha_{2}=1 / 20, \quad \alpha_{3}=1 / 15, \quad \alpha_{4}=1 / 10, \\
& \delta_{1}=1 / 20, \quad \delta_{2}=1 / 15, \quad \delta_{3}=1 / 10, \\
& \delta_{4}=1 / 5, \quad \gamma_{1}=1 / 18, \quad \gamma_{2}=1 / 9, \quad \gamma_{3}=1 / 3, \\
& \sigma_{1}=1 / 16, \quad \sigma_{1}=1 / 8, \quad \sigma_{3}=3 / 16, \quad \sigma_{4}=1 / 4, \\
& \xi_{1}=1 / 2, \quad \xi_{2}=19 / 30, \quad \xi_{3}=23 / 30, \quad \eta_{1}=17 / 30 \\
& \eta_{1}=7 / 10, \quad \eta_{3}=5 / 6 .
\end{aligned}
$$


Using the given values of the parameters in (2.3)-(2.5) and (2.12)-(2.15), we find that

$$
\begin{aligned}
& \mu_{1}=23 / 60, \quad \mu_{2}=427 / 1800, \quad \mu_{3}=139 / 4320, \\
& \Delta_{1}=97 / 60, \Delta_{2}=3173 / 1800, \quad \Delta_{3}=4181 / 4320, \\
& \Omega \simeq 1.573224, \quad \Omega_{1} \simeq 1.951384, \\
& Z_{1} \simeq 1.911201, \quad Z_{2} \simeq 3.547288 .
\end{aligned}
$$

In order to illustrate Theorem 3.6, we take

$$
f\left(t, x(t),{ }^{c} D^{\beta} x(t)\right)=\frac{(t+1)^{2}}{7^{1 / 3}}\left(\frac{1}{4}+\frac{1}{28} \sin x(t)+\frac{1}{40}{ }^{c} D^{\beta} x(t)\right) .
$$

Clearly, $\left|f\left(t, x(t),{ }^{c} D^{\beta} x(t)\right)\right| \leq m(t)\left(\psi_{1}(|x(t)|)+\psi_{2}\left(\left|{ }^{c} D^{\beta} x(t)\right|\right)\right)$ with $m(t)=\frac{(t+1)^{2}}{7^{1 / 3}},\|m\|=$ $\left(\frac{127}{49}\right)^{1 / 3}, \psi_{1}(|x(t)|)=\frac{1}{4}+\frac{|x(t)|}{28}, \psi_{2}\left(\left.\right|^{c} D^{\beta} x(t) \mid\right)=\frac{\left|D^{c} D^{\beta} x(t)\right|}{40}$. Thus condition $\left(A_{2}\right)$ is satisfied. Then, from $\left(A_{3}\right)$ together with the values of $Z_{1}$ and $Z_{2}$ given by (5.2), we find that $M>3.440883$. Consequently, the conclusion of Theorem 3.6 applies and problem (1.1)-(1.2) with (5.1) and (5.3) has at least one solution on $[0,1]$.

For the illustration of Theorem 4.5, let us take

$$
f\left(t, x(t),{ }^{c} D^{1 / 2} x(t)\right)=\frac{1}{4(t+1)^{2 / 3}}\left(\frac{1}{2}+\frac{|x|}{1+|x|}+\tan ^{-1}\left({ }^{c} D^{1 / 2} x(t)\right)\right)
$$

in (1.1) and note that

$$
\left|f\left(t, x_{1},{ }^{c} D^{1 / 2} x_{1}\right)-f\left(t, x_{2},{ }^{c} D^{1 / 2} x_{2}\right)\right| \leq \frac{1}{4(t+1)^{2 / 3}}\left(\left|x_{1}-x_{2}\right|+\left|{ }^{c} D^{1 / 2} x_{1}-{ }^{c} D^{1 / 2} x_{2}\right|\right) .
$$

Here $m(t)=\frac{1}{4(t+1)^{2 / 3}}$ with $\|m\|=\frac{1}{(384)^{1 / 3}}$. Using the values of $Z_{1}$ and $Z_{2}$ given by (5.2), we find that $\|m\|\left(Z_{1}+Z_{2}\right)=0.750981<1$. As all the conditions of Theorem 4.5 are satisfied, therefore there exists a unique solution of problem (1.1)-(1.2) with the data (5.1) and (5.4) on $[0,1]$.

Next we demonstrate the application of Corollary 4.6 by choosing a function

$$
f\left(t, x(t),{ }^{c} D^{1 / 2} x(t)\right)=L\left(\frac{1}{8}+|x|+\frac{\left|{ }^{c} D^{1 / 2} x(t)\right|}{1+\left|{ }^{c} D^{1 / 2} x(t)\right|}\right),
$$

which is clearly a Lipschitz function with Lipschitz constant $L$. Observe that the condition $L\left(\Omega+\Omega_{1}\right)<1$ is satisfied for $0<L<0.283719\left(\Omega\right.$ and $\Omega_{1}$ are given by (5.2)). Thus, by Corollary 4.6, problem (1.1)-(1.2) with data (5.1) and (5.5) has a unique solution on $[0,1]$.

\section{Conclusions}

We have studied a nonlinear fractional differential equation with nonlinearity depending on the unknown function together with its lower-order fractional derivative, equipped with a general type of non-separated boundary conditions involving finite many nonlocal points and sub-segments of the interval $[0,1]$. Several existence and uniqueness results have been derived by applying different tools of the fixed point theory. Our results are quite general and give rise to many new cases by assigning different values to the parameters involved in the problem. For explanation, we enlist some special cases. 
- By fixing $r_{j}=0=\gamma_{j}, j=1, \ldots, p$, in (1.2), our results correspond to the ones for non-separated multi-point boundary conditions. On the other hand, we obtain the results for non-separated multi-strip boundary conditions by taking $\alpha_{j}=0=\delta_{j}, j=1, \ldots, p$, in (1.2).

- In case we choose $r_{j}=\gamma_{j}=\alpha_{j}=\delta_{j}=0, j=1, \ldots, p$, in (1.2), our results correspond to periodic/anti-periodic type boundary conditions $x(0)=-(b / a) x(1), x^{\prime}(0)=-(d / c) x^{\prime}(1)$. In particular, we have the results for anti-periodic type boundary conditions when $(b / a)=1=(d / c)$. For more details on anti-periodic fractional order boundary value problems, see [28].

- Letting $a=c=1, b=d=0$, conditions (1.2) read as

$$
x(0)=\sum_{i=1}^{m-2} \alpha_{i} x\left(\sigma_{i}\right)+\sum_{j=1}^{p-2} r_{j} \int_{\xi_{j}}^{\eta_{i}} x(s) d s, \quad x^{\prime}(0)=\sum_{i=1}^{m-2} \delta_{i} x^{\prime}\left(\sigma_{i}\right)+\sum_{j=1}^{p-2} \gamma_{j} \int_{\xi_{j}}^{\eta_{j}} x^{\prime}(s) d s,
$$

which are initial nonlocal multi-point and multi-strip type conditions. Similarly (1.2) take the form of terminal nonlocal multi-point and multi-strip type conditions for $a=c=0, b=d=1$.

- If we take $a=c=b=d=0$ in (1.2), then our results correspond to nonlocal multi-point and multi-strip conditions.

In the nutshell, the boundary value problem studied in this paper is of fairly general nature and covers a variety of special cases.

\section{Acknowledgements}

This project was funded by the Deanship of Scientific Research (DSR) at King Abdulaziz University, Jeddah, under grant no. (RG-15-130-38). The authors, therefore, acknowledge with thanks DSR technical and financial support.

\section{Funding}

Not applicable.

List of abbreviations

Not applicable.

Availability of data and materials

Not applicable.

\section{Competing interests}

The authors declare that they have no competing interests.

\section{Authors' contributions}

Each of the authors, BA, SKN, AA, WS, and RPA, contributed equally to each part of this work. All authors read and approved the final manuscript.

\section{Author details}

'NAAM-Research Group, Department of Mathematics, Faculty of Science, King Abdulaziz University, Jeddah, Saudi Arabia. ${ }^{2}$ Department of Mathematics, University of loannina, Ioannina, Greece. ${ }^{3}$ Department of Mathematics, Texas A\&M University, Kingsville, USA.

\section{Publisher's Note}

Springer Nature remains neutral with regard to jurisdictional claims in published maps and institutional affiliations.

Received: 3 January 2018 Accepted: 1 March 2018 Published online: 13 March 2018

\section{References}

1. Goodrich, C.: Existence and uniqueness of solutions to a fractional difference equation with nonlocal conditions. Comput. Math. Appl. 61, 191-202 (2011)

2. Wang, J.R., Zhou, Y., Feckan, M.: On recent developments in the theory of boundary value problems for impulsive fractional differential equations. Comput. Math. Appl. 64, 3008-3020 (2012) 
3. Henderson, J., Kosmatov, N.: Eigenvalue comparison for fractional boundary value problems with the Caputo derivative. Fract. Calc. Appl. Anal. 17, 872-880 (2014)

4. Liu, Y.: Multiple positive solutions of BVPs for singular fractional differential equations with non-Caratheodory nonlinearities. Math. Model. Anal. 19, 395-416 (2014)

5. Henderson, J., Luca, R., Tudorache, A.: On a system of fractional differential equations with coupled integral boundary conditions. Fract. Calc. Appl. Anal. 18, 361-386 (2015)

6. Peng, L., Zhou, Y.: Bifurcation from interval and positive solutions of the three-point boundary value problem for fractional differential equations. Appl. Math. Comput. 257, 458-466 (2015)

7. Zhang, L., Ahmad, B., Wang, G.: Successive iterations for positive extremal solutions of nonlinear fractional differential equations on a half line. Bull. Aust. Math. Soc. 91, 116-128 (2015)

8. Ahmad, B., Ntouyas, S.K.: Some fractional-order one-dimensional semi-linear problems under nonlocal integral boundary conditions. Rev. R. Acad. Cienc. Exactas Fís. Nat., Ser. A Mat. 110, 159-172 (2016)

9. Qarout, D., Ahmad, B., Alsaedi, A.: Existence theorems for semi-linear Caputo fractional differential equations with nonlocal discrete and integral boundary conditions. Fract. Calc. Appl. Anal. 19, 463-479 (2016)

10. Nyamoradi, N., Zhou, Y: Multiple solutions for a nonlinear fractional boundary value problems via variational methods. Fixed Point Theory 17, 111-122 (2016)

11. Ahmad, B., Luca, R.: Existence of solutions for a sequential fractional integro-differential system with coupled integral boundary conditions. Chaos Solitons Fractals 104, 378-388 (2017)

12. Alsulami, H.H., Ntouyas, S.K., Agarwal, R.P., Ahmad, B., Alsaedi, A.: A study of fractional-order coupled systems with a new concept of coupled non-separated boundary conditions. Bound. Value Probl. 2017, 68 (2017)

13. Agarwal, R.P., Ahmad, B., Garout, D., Alsaedi, A.: Existence results for coupled nonlinear fractional differential equations equipped with nonlocal coupled flux and multi-point boundary conditions. Chaos Solitons Fractals 102, 149-161 (2017)

14. Zhang, H.: Nonlocal boundary value problems of fractional order at resonance with integral conditions. Adv. Differ. Equ. 2017, 326 (2017)

15. Sayevand, K., Pichaghchi, K.: Efficient algorithms for analyzing the singularly perturbed boundary value problems of fractional order. Commun. Nonlinear Sci. Numer. Simul. 57, 136-168 (2018)

16. Agarwal, R.P., Alsaedi, A., Alsharif, A., Ahmad, B.: On nonlinear fractional-order boundary value problems with nonlocal multi-point conditions involving Liouville-Caputo derivative. Differ. Equ. Appl. 9, 147-160 (2017)

17. Ntouyas, S.K., Tariboon, J.: Fractional boundary value problems with multiple orders of fractional derivatives and integrals. Electron. J. Differ. Equ. 2017, 100 (2017)

18. Ahmad, B., Ntouyas, S.K., Tariboon, J., Alsaedi, A.: A study of nonlinear fractional-order boundary value problem with nonlocal Erdélyi-Kober and generalized Riemann-Liouville type integral boundary conditions. Math. Model. Anal. 22, $121-139(2017)$

19. Bitsadze, A., Samarskii, A.: On some simple generalizations of linear elliptic boundary problems. Russ. Acad. Sci. Dokl. Math. 10, 398-400 (1969)

20. Ahmad, B., Alsaedi, A., Alghamdi, B.S.: Analytic approximation of solutions of the forced Duffing equation with integral boundary conditions. Nonlinear Anal., Real World Appl. 9, 1727-1740 (2008)

21. Čiegis, R., Bugajev, A.: Numerical approximation of one model of the bacterial self-organization. Nonlinear Anal., Model. Control 17, 253-270 (2012)

22. Kilbas, A.A., Srivastava, H.M., Trujillo, J..: Theory and Applications of Fractional Differential Equations. North-Holland Mathematics Studies, vol. 204. Elsevier Science B.V., Amsterdam (2006)

23. Podlubny, I.: Fractional Differential Equations. Academic Press, San Diego (1999)

24. Su, X.: Boundary value problem for a coupled system of nonlinear fractional differential equations. Appl. Math. Lett. $22,64-69(2009)$

25. Granas, A., Dugundji, J.: Fixed Point Theory. Springer, New York (2003)

26. Krasnoselskii, M.A.: Two remarks on the method of successive approximations. Usp. Mat. Nauk 10, 123-127 (1955)

27. Boyd, D.W., Wong, J.S.W.: On nonlinear contractions. Proc. Am. Math. Soc. 20, $458-464$ (1969)

28. Agarwal, R.P., Ahmad, B., Alsaedi, A.: Fractional-order differential equations with anti-periodic boundary conditions: a survey. Bound. Value Probl. 2017, 173 (2017)

\section{Submit your manuscript to a SpringerOpen ${ }^{\circ}$ journal and benefit from:}

- Convenient online submission

- Rigorous peer review

- Open access: articles freely available online

- High visibility within the field

- Retaining the copyright to your article

Submit your next manuscript at $\boldsymbol{~ s p r i n g e r o p e n . c o m ~}$ 